\title{
Comparisons of molecular diversity indices, selective sweeps and population structure of African rice with its wild progenitor and Asian rice
}

\author{
Marie Noelle Ndjiondjop ${ }^{1}$ - Nikolaos Alachiotis ${ }^{2}$. Pavlos Pavlidis ${ }^{2}$. Alphonse Goungoulou ${ }^{1}$. \\ Sèdjro Bienvenu Kpeki ${ }^{1} \cdot$ Dule Zhao $^{1} \cdot$ Kassa Semagn $^{1}$
}

Received: 11 October 2018 / Accepted: 11 December 2018 / Published online: 21 December 2018

(c) The Author(s) 2018

\begin{abstract}
Key message The extent of molecular diversity parameters across three rice species was compared using large germplasm collection genotyped with genomewide SNPs and SNPs that fell within selective sweep regions.

Abstract Previous studies conducted on limited number of accessions have reported very low genetic variation in African rice (Oryza glaberrima Steud.) as compared to its wild progenitor (O. barthii A. Chev.) and to Asian rice (O. sativa L.). Here, we characterized a large collection of African rice and compared its molecular diversity indices and population structure with the two other species using genomewide single nucleotide polymorphisms (SNPs) and SNPs that mapped within selective sweeps. A total of 3245 samples representing African rice (2358), Asian rice (772) and $O$. barthii (115) were genotyped with 26,073 physically mapped SNPs. Using all SNPs, the level of marker polymorphism, average genetic distance and nucleotide diversity in African rice accounted for $59.1 \%, 63.2 \%$ and $37.1 \%$ of that of $O$. barthii, respectively. SNP polymorphism and overall nucleotide diversity of the African rice accounted for 20.1-32.1 and 16.3-37.3\% of that of the Asian rice, respectively. We identified 780 SNPs that fell within 37 candidate selective sweeps in African rice, which were distributed across all 12 rice chromosomes. Nucleotide diversity of the African rice estimated from the 780 SNPs was $8.3 \times 10^{-4}$, which is not only 20 -fold smaller than the value estimated from all genomewide SNPs $\left(\pi=1.6 \times 10^{-2}\right)$, but also accounted for just $4.1 \%, 0.9 \%$ and $2.1 \%$ of that of $O$. barthii, lowland Asian rice and upland Asian rice, respectively. The genotype data generated for a large collection of rice accessions conserved at the AfricaRice genebank will be highly useful for the global rice community and promote germplasm use.
\end{abstract}

Communicated by Yunbi Xu.

Electronic supplementary material The online version of this article (https://doi.org/10.1007/s00122-018-3268-2) contains supplementary material, which is available to authorized users.

Marie Noelle Ndjiondjop

m.ndjiondjop@cgiar.org

Kassa Semagn

k.semagn@gmail.com

1 M'bé Research Station, Africa Rice Center (AfricaRice), 01 B.P. 2551, Bouaké 01, Côte d'Ivoire

2 Institute of Computer Science, Foundation for Research and Technology-Hellas, Nikolaou Plastira 100, 70013 Heraklion, Crete, Greece

\section{Introduction}

Rice (Oryza L.) is one of the top three food crops in the world, ranked second in total area harvested and production per hectare and third in total global production after maize and wheat (http://www.fao.org/faostat/en/\#data; accessed in Dec. 2018). The genus Oryza consists of 27 species and 11 genome types (Stein et al. 2018). Eight species encompassing five genomes are found on the African continent, which include the AA (Oryza barthii A. Chev., O. glaberrima Steud., $O$. sativa L., and Oryza longistaminata Chev. and Röhr), BB (O. punctata), CC (O. eichingeri), FF (O. brachyantha) and $\mathrm{BBCC}(O$. schweinfurthiana) genome species (Vaughan et al. 2003). All these species are conserved at the AfricaRice genebank, each with a varying number of accessions ranging from few to several thousands. Of all the $\sim 22,000$ registered rice samples conserved at the AfricaRice genebank, about $14 \%$ belong to African rice $(O$. 
glaberrima), $85 \%$ to Asian rice (O. sativa) and $1 \%$ of the other wild species.

African and Asian rice have been shown to be independently domesticated from the wild species of $O$. barthii in Africa and O. rufipogon in Asia, respectively (Wang et al. 2014). Recent studies of the genetic variation, relatedness and population structure of 2179 African rice accessions indicated that overall level of polymorphism and genetic distance between pairs of accessions observed across this large number of samples were very low (Ndjiondjop et al. 2017). Other studies compared the extent of genetic variation of African rice using whole genome sequencing and targeted sequencing of a few genes that have undergone selection $(\mathrm{Li}$ et al. 2011; Nabholz et al. 2014; Wang et al. 2014; Meyer et al. 2016; Win et al. 2017; Cubry et al. 2018; Lv et al. 2018) and reported very low genetic variation in African rice as compared to Asian rice and to its wild progenitor $O$. barthii. Results of the gene-based sequencing studies suggest a strong domestication bottleneck, while those of genomewide studies suggested the role of both genetic bottleneck and selective sweeps as the main factors for the lower genetic diversity observed in the cultivated African rice compared to its wild progenitor (Vaughan et al. 2008). However, all previous studies that compared the extent of nucleotide diversity and effect of selective sweeps in African rice and $O$. barthii were based on small number of accessions, ranging from 9 to 163 and from 10 to 88 samples, respectively. Nucleotide diversity in such a small sample may be depressed more based on the sample size itself, than on evolutionary forces acting on the entire species, which forms one of the bases of this study.

Selection (selective) sweeps, which refer to a reduction in nucleotide diversity near advantageous mutations in useful genes, leave distinct signatures in genomes; this enables the detection of loci that have undergone positive selection (Peter et al. 2012; Chen et al. 2016; Alachiotis and Pavlidis 2018). Positive selection increases the frequency of a beneficial allele within a population and may even lead to fixation. While this process increases fitness of the individuals carrying the beneficial allele, it reduces overall genetic diversity in a population or species until recombination and mutation introduce new alleles in these selected regions (Olsen et al. 2006; Vitti et al. 2013; Alachiotis and Pavlidis 2016). For some time, however, the selected alleles are present at high frequency, while the newly introduced alleles (or residual, less beneficial alleles) are found at low frequency, and may remain so unless they are neutral or beneficial in effect. Using data from small number of samples, several studies reported a reduction in genetic diversity in domesticated (cultivated) crops as compared to their wild progenitors (Reif et al. 2005; Wright et al. 2005; Gore et al. 2009; Lam et al. 2010; Ding et al. 2011; Huang et al. 2012; Yuan et al. 2017), but those results may also be biased by samples sizes. Thus, assessment of the level of nucleotide diversity in large number of individuals using both genomewide markers and a subset of markers that physically map within selective sweep regions would provide confidence, which forms another basis of this study.

A wide range of statistical methods have been used to identify traces of intra-species selective sweeps by detecting regions of reduced genetic variation, which have undergone a selective sweep (Crisci et al. 2013; Alachiotis and Pavlidis 2016; Pavlidis and Alachiotis 2017). Using simulated data, Crisci and colleagues evaluated SweepFinder (Nielsen et al. 2005), SweeD (Pavlidis et al. 2013), OmegaPlus (Alachiotis et al. 2012) and iHS (Voight et al. 2006) in terms of efficiency on type I and II errors, effect of population structure and size and genome coverage. Overall, OmegaPlus performed better than the other three methods. In the present study, we used DArTseq genotyping of 3245 samples representing African rice, Asian rice and $O$. barthii to (1) identify genomic regions that have undergone selective sweeps in African rice and examined if those selective sweeps showed greater reduction in nucleotide diversity in African rice as compared with its wild relative $O$. barthii and Asian rice and (2) compare the extent of genetic relatedness and population structure of the three rice species using genomewide SNPs.

\section{Materials and methods}

This study was conducted on a total of 3245 accessions and varieties (hereafter referred to as samples) conserved at the AfricaRice genebank (Supplementary Table S1), which represent $O$. barthii (115 samples), African rice (2358 samples) and Asian rice (772 samples). About $92 \%$ of the African rice (Ndjiondjop et al. 2017) and 43\% of the Asian rice (Ndjiondjop et al. 2018a) samples had been previously used for genetic diversity and population structure studies conducted within each species. Nearly, all samples had also been used for identification of species- and subspecies-diagnostic SNPs for routine genotyping quality control analysis to minimize errors during germplasm collection, acquisition and routine genebank operations (Ndjiondjop et al. 2018b). The detailed procedures for genomic DNA extraction and SNP genotyping using DArTseq ${ }^{\mathrm{TM}}$ were described in our previous study (Ndjiondjop et al. 2017). Each sample was genotyped with 31,739 SNPs by DArT Pty Ltd, Australia (http://www.diver sityarrays.com) of which 26,073 SNPs (Dataset-1) were physically mapped on to the 12 rice chromosomes and had two alleles each irrespective of their minor allele frequency (Table 1). SNPs that were not physically mapped (5606 SNPs) or SNPs that were physically mapped but were completely monomorphic across all samples (60 SNPs) were excluded from all statistical analyses. Of the 26,073 SNPs in Dataset-1, nearly 89\% (23,079 SNPs) were polymorphic 


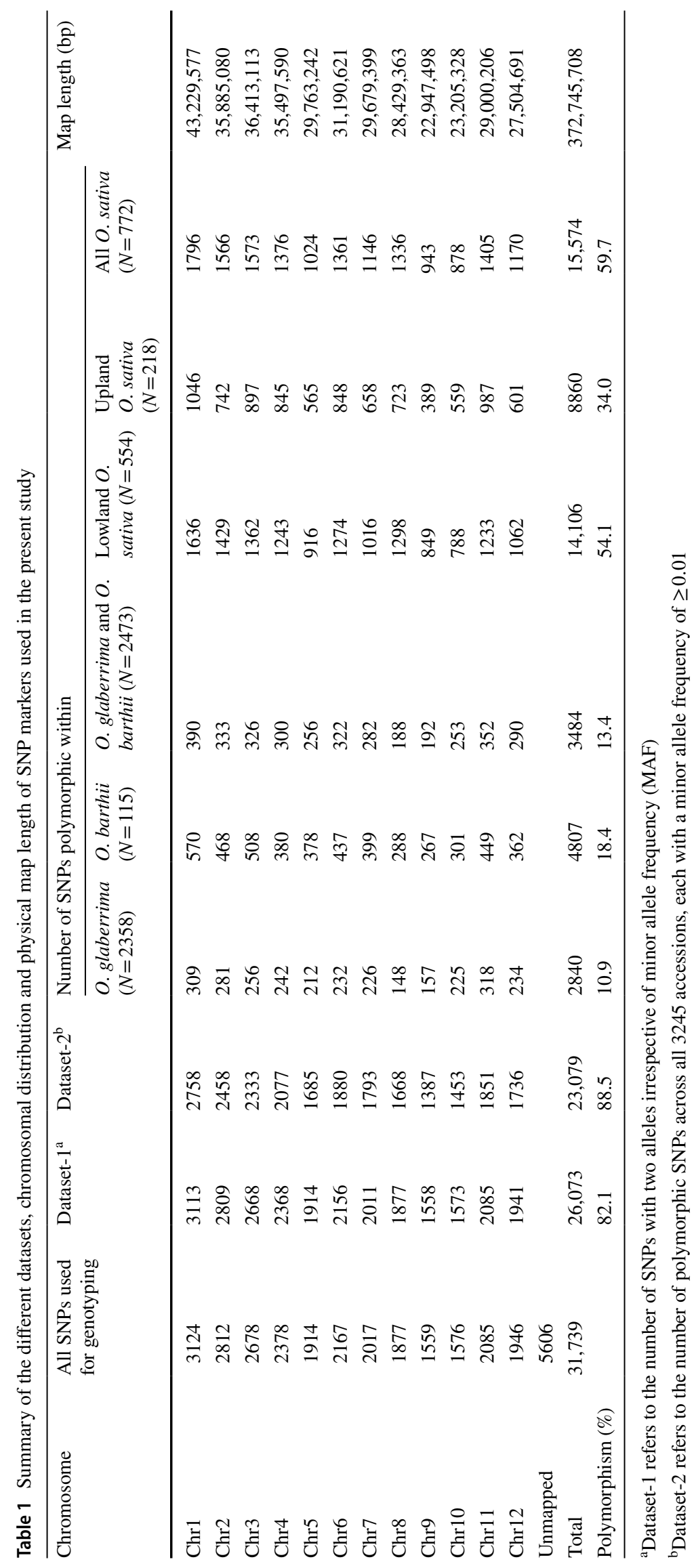


across all 3245 samples (Dataset-2), each having a minor allele frequency ranging from 0.01 to 0.496 (Supplementary Table S2).

Hapmap input files from TASSEL v.5.2.48 were exported to PHYLIP interleaved format, which were then converted to both MEGA X (Kumar et al. 2016) and ARLEQUIN v.3.5.2.2 (Excoffier and Lischer 2010) formats using PGDSpider (Lischer and Excoffier 2012). We used MEGA X (Kumar et al. 2016) to estimate number of segregating sites, proportion of polymorphic sites $(\mathrm{Ps})$, Theta $\left(\theta_{\mathrm{S}}\right)$, nucleotide diversity $(\theta \pi)$ and Tajima's $D$ test statistic (Tajima 1989). Tajima's $D$ was used to test the null hypothesis of selective neutrality in each species and groups observed within species. To assess the effect of sample size on molecular diversity indices of African rice, we conducted the analyses on (1) a minicore of 350 accessions (Ndjiondjop et al. 2017); (2) subsets of 115 and 163 randomly chosen accessions from the 350 minicore set to get the same sample size as the $O$. barthii used in the current study and that of a recent study (Cubry et al. 2018), respectively, and (3) randomly selected samples of 115 African rice accessions to get the same number of samples as that of $O$. barthii in the present study, which was repeated 20 times. Finally, two additional analyses were also run on two sample sizes of 983 and 1375 African rice accessions proposed based on neighbor-joining cluster and principal component analyses (Supplementary Fig. S1).

OmegaPlus v.3.0.2 (Alachiotis et al. 2012) was used to detect selective sweeps that may have undergone positive selection by dividing Dataset- 1 into four subsets that corresponded to African rice, O. barthii, lowland O. sativa, which are primarily indica, and upland $O$. sativa, which are primarily japonica. $O$. sativa samples were divided into lowland and upland ecologies for some statistical analyses due to the distinct population structure observed between these two ecological groups based on results from neighbor-joining cluster analysis (Supplementary Fig. S2) and principal component analysis (Fig. 1). Each subset of data for OmegaPlus was then converted into reference and alternative alleles using the variant call format (VCF) file conversion option in TASSEL v.5.2.48, which refers to the major and minor alleles, respectively. OmegaPlus v.3.0.2 was separately executed on each subset of data as described in a previous study (Alachiotis and Pavlidis 2016), evaluating a grid of 10,000 equidistant physical locations along each subset (Alachiotis and Pavlidis 2018). The average distance between any two consecutive locations was $3105 \mathrm{bp}$, while each score computation entails the exhaustive evaluation of 1-Mb overlapping windows. The threshold score for declaring selective sweeps to be significant was set as the 99 th percentile, so the $1 \%$ with the highest scores was retained to represent a candidate selective sweep region. Two or more selective sweeps

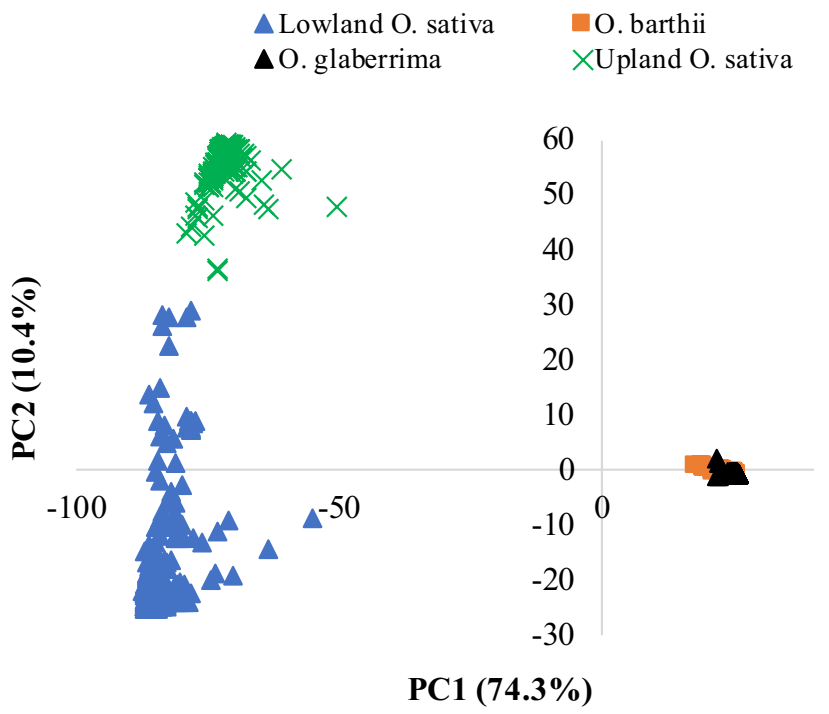

Fig. 1 Plot of PC1 and PC2 from principal component analysis of 3245 samples representing Oryza glaberrima (2358), O. barthii (115) and $O$. sativa (772) using 23,079 polymorphic SNPs. As only $14.4 \%$ of the 23,079 SNPs were polymorphic between $O$. barthii and $O$. glaberrima, these two species appeared nearly identical, while $O$. sativa genotypes showed clear separation into upland (218) and lowland (554) ecologies

with an overlapping start and/or end position were considered the same candidate selective sweep. To minimize the length of the manuscript, only peaks (1) that were specific to African rice, (2) had at least three adjacent SNPs within the start and end positions (interval), and (3) had likelihood test scores greater than the minimum threshold value were chosen for further analyses. The start and end physical positions of each selective sweep region were used to search for candidate genes at the Gramene Genome Brower (http://ensembl.gramene.org/genome_browser/ index.html).

Additional subsets of genotypic data consisting of all SNPs that mapped across all selective sweeps detected in African rice (Dataset-3), O. barthii (Dataset-4), lowland $O$. sativa (Dataset-5) and upland O. sativa (Dataset-6) were also used to compute nucleotide diversity in MEGA X (Kumar et al. 2016). Identity-by-state (IBS)-based genetic distance matrix, neighbor-joining cluster analysis, principal component analysis (PCA), analysis of molecular variance (Excoffier et al. 1992) and $\mathrm{F}_{\mathrm{ST}}$-based pairwise genetic distance matrices (Holsinger and Weir 2009) were conducted by filtering the genotypic data with a minor allele frequency (MAF) of $\geq 0.01$ as described in our previous papers (Ndjiondjop et al. 2017, 2018a). The genotypic data used for these analyses include the 23,079 SNPs in Dataset-2 plus other species and group-specific genotypic files created from Dataset-1 by filtering with MAF of $\geq 0.01$. 


\section{Results}

\section{Test for selective neutrality and detection of selective sweeps}

Table 2 summarizes the different evolutionary parameters estimated across the three species. Tajima's $D$ value computed from the 26,073 SNPs in Dataset-1 was positive in the wild $O$. barthii $(0.219)$, but negative in the cultivated African $(-1.381)$ and Asian rice $(-0.257$ for lowland and -1.513 for upland $O$. sativa). The negative Tajima's $D$ values observed in the two cultivated rice species are indicative of positive selection and reject the null hypothesis that all mutations are selectively neutral. Using OmegaPlus, we identified a total of 37 candidate selective sweep regions in African rice (Fig. 2), 89 regions in lowland Asian rice and 68 regions in upland Asian rice (Table 3, Supplementary Fig. S3). The candidate selective sweeps detected in African rice spanned from 28 to $850 \mathrm{~kb}$ and consisted of clusters of markers that varied from 11 to 74 SNPs (Supplementary Table S3); the number of selective sweeps detected per chromosome ranged from 1 on chromosome 3 to 6 on chromosome 2 (Table 3). Overall, there were a total of 780 SNPs that mapped within the 37 candidate selective sweeps detected in African rice (Dataset-3). Only four of the 780 SNPs had MAF $\geq 0.01$ in African rice as compared to 88 SNPs in $O$. barthii, 419 SNPs in lowland Asian rice and 275 SNPs in upland Asian rice (Supplementary Table S2). Selective sweeps increase the frequency of beneficial alleles and surrounding variants and may eventually lead to fixation, and recombination and mutation that introduce new alleles are rare (causing alleles of very low frequency), which are evident in Supplementary Table $\mathrm{S} 2$.

Selective sweeps were more common in Asian rice than in African rice (Table 3). Each selective sweep identified in lowland and upland Asian rice spanned from 55 to $771 \mathrm{~kb}$ and from 47 to $1045 \mathrm{~kb}$, respectively, and harbored clusters of SNPs ranging from 6 to 54 and from 10 to 76 markers (Supplementary Table S3). Five of the 89 selective sweeps identified in lowland Asian rice (3: 17,580,209-17,811,736, 5: 15,191,088-15,198,145, 7: $7,420,481-7,526,572,9: 15,703,948-16,396,541$ and 11: $16,030,588-16,164,430)$ and seven of the selective sweeps in upland Asian rice $(1: 4,537,132-4,724,160$, 2: 6,069,007-6,374,310, 2: 21,994,604-22,316,127, 8: $23,755,429-23,755,429,9: 15,547,000-15,556,789,11$ : 442,387-442,387 and 11: 22,278,851-22,445,600) were partial overlapping with selective sweeps detected in African rice (Supplementary Tables S2, S3). Although the positive Tajima's $D$ value in $O$. barthii supports the null hypothesis of selective neutrality, results of
OmegaPlus revealed 36 selective sweeps in this wild species of which six regions $(3: 17,716,633-17,811,736$, 5: 13,940,602-14,087,325, 9: 16,021,480-16,396,541, 10: $3,705,613-3,806,404,11: 442,387-595,142$ and 11: $22,228,554-22,267,775)$ were shared with those detected in African rice (Supplementary Tables S2, S3). Overall, we identified a total 1353 SNPs, 1426 SNPs and 1421 SNPs that fell within the selective sweeps detected in $O$. barthii, lowland and upland Asian rice, respectively, which were used for computing molecular diversity indices (see below).

\section{Candidate genes in selective sweeps detected in African rice}

To gain insight into possible roles of each selective sweep in African rice, we compiled a list of 901 protein coding candidate genes found within the 37 selected regions (Supplementary Table S4). There were two to ninety-two genes per selective sweep region of which some had known functions, descriptions of which can be found in Supplementary Table S3. As shown in the Manhattan plot in Fig. 2, the highest test scores $\left(1.0 \times 10^{5}-28.6 \times 10^{5}\right)$ were observed in $850 \mathrm{~kb}$ interval on chromosome $9(9: 15,547,000-16,396,541)$. This region harbored clusters of 87 protein coding genes (Supplementary Table S4), including DEEPER ROOTING 1 (DRO1), that play role for adapting to the dry sub-Saharan region and DELAYED SEED GERMINATION 1 (OsDSG1) that prevent germination when there is insufficient moisture in the soil and under storage conditions. The role of other candidate genes with known function has been summarized in Supplementary Table S3 and described in detail in the discussion section.

\section{Molecular diversity indices}

Of the 26,073 SNPs in Dataset-1, the number of segregating sites (6843) and proportion of polymorphic sites (0.262) in African rice were nearly $20 \%$ greater than those of $O$. barthii, but $64.2 \%$ and $46.3 \%$ smaller than those of the lowland and upland Asian rice, respectively (Table 2). Theta $(\Theta)$ in African rice was 0.031 , which is $76.7 \%$ of that of O. barthii (0.041), $29.6 \%$ of the lowland and $38.4 \%$ of the upland Asian rice. Nucleotide diversity $(\pi)$ in African rice was 0.016 , which accounted for $37.1 \%, 16.7 \%$ and $37.3 \%$ of the values observed in $O$. barthii (0.044), lowland (0.097) and upland (0.043) Asian rice, respectively. The number of segregating sites, proportion of polymorphic sites and $\Theta$ and $\pi$ values computed from the 23,079 SNPs in Dataset- 2 that were polymorphic across all 3245 samples at a MAF $\geq 0.01$ were similar to that of Dataset-1.

Using genotype data of the 780 SNPs (Dataset-3) that fell within the 37 selective sweeps detected in African rice, 
Table 2 Summary of the molecular diversity indices of three rice species based on six datasets of different marker density

\begin{tabular}{|c|c|c|c|c|c|c|}
\hline \multirow[t]{2}{*}{ Species and dataset ${ }^{\mathrm{a}}$} & \multicolumn{6}{|c|}{ Results from Tajima's neutrality test ${ }^{\mathrm{b}}$} \\
\hline & $m$ & $S$ & Ps & $\theta_{\mathrm{S}}$ & $\theta \pi$ & $D$ \\
\hline \multicolumn{7}{|l|}{ All $O$. glaberrima } \\
\hline Dataset-1 & 2358 & 6843 & 0.262 & 0.031 & 0.01620 & -1.381 \\
\hline Dataset-2 & 2358 & 6057 & 0.262 & 0.031 & 0.01806 & -1.213 \\
\hline Dataset-3 & 2358 & 158 & 0.203 & 0.024 & 0.00083 & -2.641 \\
\hline Dataset-4 & 2358 & 320 & 0.272 & 0.033 & 0.01247 & -1.725 \\
\hline Dataset-5 & 2358 & 416 & 0.292 & 0.035 & 0.02194 & -1.045 \\
\hline Dataset-6 & 2358 & 374 & 0.263 & 0.032 & 0.01391 & -1.565 \\
\hline \multicolumn{7}{|c|}{ Group-1 O. glaberrima } \\
\hline Dataset-1 & 983 & 4427 & 0.170 & 0.023 & 0.01425 & -1.101 \\
\hline Dataset-2 & 983 & 4108 & 0.178 & 0.024 & 0.01592 & -0.979 \\
\hline Dataset-3 & 983 & 76 & 0.097 & 0.013 & 0.00070 & -2.598 \\
\hline Dataset-4 & 983 & 204 & 0.174 & 0.023 & 0.01378 & -1.169 \\
\hline Dataset-5 & 983 & 261 & 0.183 & 0.025 & 0.01866 & -0.690 \\
\hline Dataset-6 & 983 & 246 & 0.173 & 0.023 & 0.01423 & -1.115 \\
\hline \multicolumn{7}{|c|}{ Group-2 O. glaberrima } \\
\hline Dataset-1 & 1375 & 5414 & 0.208 & 0.027 & 0.01499 & -1.270 \\
\hline Dataset-2 & 1375 & 4834 & 0.209 & 0.027 & 0.01664 & -1.106 \\
\hline Dataset-3 & 1375 & 106 & 0.136 & 0.017 & 0.00089 & -2.612 \\
\hline Dataset-4 & 1375 & 244 & 0.208 & 0.027 & 0.00944 & -1.832 \\
\hline Dataset-5 & 1375 & 354 & 0.248 & 0.032 & 0.02054 & -1.014 \\
\hline Dataset-6 & 1375 & 285 & 0.201 & 0.026 & 0.01176 & -1.546 \\
\hline \multicolumn{7}{|c|}{ Minicore $O$. glaberrima } \\
\hline Dataset-1 & 350 & 4664 & 0.179 & 0.028 & 0.01870 & -1.020 \\
\hline Dataset-2 & 350 & 4061 & 0.176 & 0.027 & 0.02066 & -0.763 \\
\hline Dataset-3 & 350 & 78 & 0.100 & 0.016 & 0.00106 & -2.733 \\
\hline Dataset-4 & 350 & 198 & 0.169 & 0.026 & 0.01473 & -1.331 \\
\hline Dataset-5 & 350 & 294 & 0.206 & 0.032 & 0.02453 & -0.719 \\
\hline Dataset-6 & 350 & 265 & 0.186 & 0.029 & 0.01720 & -1.244 \\
\hline \multicolumn{7}{|c|}{ Subset of minicore $O$. glaberrima } \\
\hline Dataset-1 & 163 & 4114 & 0.158 & 0.028 & 0.01948 & -0.986 \\
\hline Dataset-2 & 163 & 3632 & 0.157 & 0.028 & 0.02147 & -0.744 \\
\hline Dataset-3 & 163 & 54 & 0.069 & 0.012 & 0.00132 & -2.716 \\
\hline Dataset-4 & 163 & 179 & 0.152 & 0.027 & 0.01655 & -1.232 \\
\hline Dataset-5 & 163 & 265 & 0.186 & 0.033 & 0.02544 & -0.724 \\
\hline Dataset-6 & 163 & 231 & 0.163 & 0.029 & 0.01809 & -1.190 \\
\hline \multicolumn{7}{|c|}{ Subset of minicore $O$. glaberrima } \\
\hline Dataset-1 & 115 & 3904 & 0.150 & 0.028 & 0.02006 & -0.971 \\
\hline Dataset-2 & 115 & 3491 & 0.151 & 0.028 & 0.02211 & -0.751 \\
\hline Dataset-3 & 115 & 54 & 0.069 & 0.013 & 0.00162 & -2.756 \\
\hline Dataset-4 & 115 & 174 & 0.148 & 0.028 & 0.01790 & -1.180 \\
\hline Dataset-5 & 115 & 244 & 0.171 & 0.032 & 0.02622 & -0.615 \\
\hline Dataset-6 & 115 & 218 & 0.153 & 0.029 & 0.01872 & -1.164 \\
\hline \multicolumn{7}{|l|}{ O. barthii } \\
\hline Dataset-1 & 115 & 5685 & 0.218 & 0.041 & 0.04366 & 0.219 \\
\hline Dataset-2 & 115 & 4919 & 0.213 & 0.040 & 0.04396 & 0.326 \\
\hline Dataset-3 & 115 & 108 & 0.138 & 0.026 & 0.02020 & -0.731 \\
\hline Dataset-4 & 115 & 254 & 0.216 & 0.041 & 0.02258 & -1.478 \\
\hline Dataset-5 & 115 & 370 & 0.259 & 0.049 & 0.05148 & 0.184 \\
\hline Dataset-6 & 115 & 317 & 0.223 & 0.042 & 0.04903 & 0.563 \\
\hline
\end{tabular}


Table 2 (continued)

\begin{tabular}{|c|c|c|c|c|c|c|}
\hline \multirow[t]{2}{*}{ Species and dataset ${ }^{\mathrm{a}}$} & \multicolumn{6}{|c|}{ Results from Tajima's neutrality test ${ }^{\mathrm{b}}$} \\
\hline & $m$ & $S$ & Ps & $\theta_{\mathrm{S}}$ & $\theta \pi$ & $D$ \\
\hline \multicolumn{7}{|c|}{ O. sativa spp. indica (lowland) } \\
\hline Dataset-1 & 554 & 19,090 & 0.732 & 0.106 & 0.09722 & -0.257 \\
\hline Dataset-2 & 554 & 17,327 & 0.751 & 0.109 & 0.10654 & -0.066 \\
\hline Dataset-3 & 554 & 585 & 0.750 & 0.109 & 0.08782 & -0.581 \\
\hline Dataset-4 & 554 & 819 & 0.697 & 0.101 & 0.09336 & -0.232 \\
\hline Dataset-5 & 554 & 856 & 0.600 & 0.087 & 0.06371 & -0.810 \\
\hline Dataset-6 & 554 & 1015 & 0.714 & 0.104 & 0.09803 & -0.163 \\
\hline \multicolumn{7}{|c|}{$O$. sativa spp. japonica (upland) } \\
\hline Dataset-1 & 218 & 12,734 & 0.488 & 0.082 & 0.04340 & -1.513 \\
\hline Dataset-2 & 218 & 12,066 & 0.523 & 0.088 & 0.04751 & -1.474 \\
\hline Dataset-3 & 218 & 398 & 0.510 & 0.086 & 0.04041 & -1.679 \\
\hline Dataset-4 & 218 & 607 & 0.517 & 0.087 & 0.04355 & -1.588 \\
\hline Dataset-5 & 218 & 604 & 0.424 & 0.071 & 0.04088 & -1.356 \\
\hline Dataset-6 & 218 & 426 & 0.299 & 0.050 & 0.01159 & -2.447 \\
\hline \multicolumn{7}{|c|}{ Both lowland and upland $O$. sativa } \\
\hline Dataset-1 & 772 & 20,642 & 0.792 & 0.110 & 0.17332 & -5.807 \\
\hline Dataset-2 & 772 & 18,763 & 0.813 & 0.113 & 0.19298 & -6.165 \\
\hline Dataset-3 & 772 & 654 & 0.838 & 0.116 & 0.18026 & 1.639 \\
\hline Dataset-4 & 772 & 910 & 0.774 & 0.107 & 0.17936 & 1.999 \\
\hline Dataset-5 & 772 & 961 & 0.674 & 0.093 & 0.13485 & 1.324 \\
\hline Dataset-6 & 772 & 1067 & 0.751 & 0.104 & 0.15331 & 1.413 \\
\hline
\end{tabular}

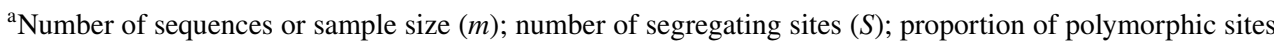
$(\mathrm{Ps})=S / n) ; \theta_{\mathrm{S}}=$ Ps/a1; nucleotide diversity $(\theta \pi)$; Tajima test $(D)$

${ }^{\mathrm{b}}$ Dataset-1 = 26,073 SNPs; Dataset-2=23,079 SNPs; Dataset-2=780 SNPs; Dataset-4=1175 SNPs; Dataset- $5=1426$ SNPs; Dataset- $6=1421$ SNPs. See Table 1 for details

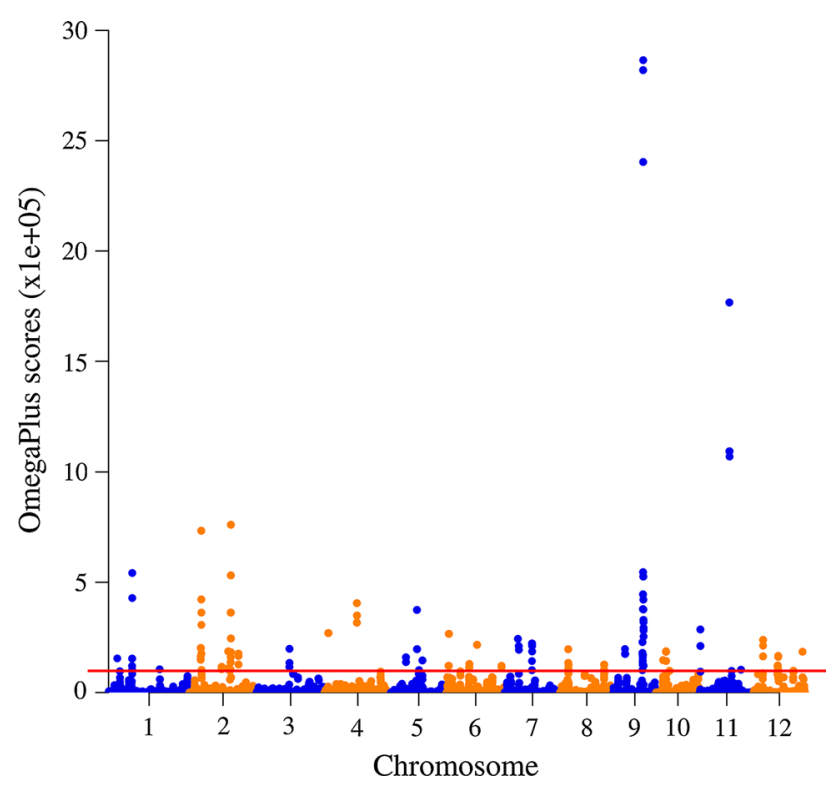

Fig. 2 Manhattan plots showing selective sweep regions detected in African rice $(O$. glaberrima). The horizontal solid line indicates the threshold value for declaring candidate selective sweeps (see material and method section for details)
Table 3 Chromosomal distribution of number of selective sweep regions identified in three rice species

\begin{tabular}{llccc}
\hline Chromosome & O. glaberrima & $\begin{array}{c}\text { Lowland } \\
\text { O. sativa }\end{array}$ & $\begin{array}{l}\text { Upland } \\
\text { O. sativa }\end{array}$ & O. barthii \\
\hline 1 & 3 & 11 & 8 & 6 \\
2 & 6 & 11 & 6 & 1 \\
3 & 1 & 15 & 5 & 2 \\
4 & 2 & 9 & 10 & 3 \\
5 & 4 & 5 & 8 & 3 \\
6 & 5 & 10 & 4 & 2 \\
7 & 3 & 8 & 4 & 1 \\
8 & 2 & 2 & 3 & 1 \\
9 & 2 & 4 & 10 & 2 \\
10 & 2 & 3 & 5 & 4 \\
11 & 3 & 5 & 3 & 6 \\
12 & 4 & 6 & 2 & 5 \\
Grand total & 37 & 89 & 68 & 36 \\
\hline
\end{tabular}

we compared the changes in nucleotide diversity across the three species. Nucleotide diversity in African rice was $8.3 \times 10^{-4}$, which accounted for just $4.1 \%$ of that of $O$. 
barthii, $0.9 \%$ and $2.1 \%$ of the lowland and upland Asian rice, respectively (Table 2). On the other hand, nucleotide diversity of the African rice computed from SNPs that fell within the selective sweeps identified in $O$. barthii (Dataset-4) and lowland Asian rice (Dataset-5) accounted for $55.2 \%$ and $34.4 \%$ of that of $O$. barthii and lowland Asian rice, respectively, which are even higher than the values obtained from the entire 26,073 SNPs in Dataset-1. These results demonstrate a more severe reduction in genetic variation in African rice due to selective sweeps detected in this species than the selective sweeps detected in the other two species. The nucleotide diversity of African rice as calculated from SNPs in selective sweeps identified in upland Asian rice (Dataset-6) was sevenfold greater than the value obtained in Dataset-3, but smaller than that of Dataset-1. The latter is not surprising due to not only much smaller Tajima $D$ value observed in upland than the lowland Asian rice (Table 2), but also the presence of more common selective sweeps between the African rice and the upland Asian rice (Supplementary Table S2). Both African rice and $O$. barthii showed reduction in nucleotide diversity in Dataset-3 than Dataset-1, but the reduction in the former was 19-fold $\left(\pi=1.6 \times 10^{-2}\right.$ in Dataset-1 vs. $8.3 \times 10^{-4}$ in Dataset- 3$)$ as compared to just twofold in $O$. barthii $\left(\pi=4.4 \times 10^{-2}\right.$ in Dataset-1 vs. $2.0 \times 10^{-2}$ in Dataset-3). On the contrary, the changes in nucleotide diversity in lowland and upland Asian rice were very minimal irrespective of the datasets; the only exceptions were Dataset-5 in the lowland Asian rice and Dataset-6 in the upland Asian rice that showed about from two to threefold smaller nucleotide diversity values as compared to the whole 26,073 SNPs.

To assess the effect of sample size on the molecular diversity indices in African rice, we repeated the analyses on five additional samples sizes $(N=115,163,350,983$ and 1375) using the 26,073 SNPs. Nucleotide diversity of African rice computed from these five smaller sample sizes accounted from 30.5 to $45.9 \%$ of that of $O$. barthii, from 13.5 to $20.6 \%$ of that of lowland Asian rice and from 30.1 to $46.2 \%$ of that of upland Asian rice (Table 2, Supplementary Table S5). Overall, nucleotide diversity values estimated from smaller sample sizes showed an increase or decrease up to $8.9 \%$ as compared to the entire 2358 accessions; hence, the severe reduction in nucleotide diversity observed in African rice than both its wild progenitor and Asian rice was very consistent irrespective of sample size (Supplementary Fig. S4) and marker density.

\section{Genetic relatedness and population structure}

Genetic distance between pairs of all 3245 samples computed from the 23,079 SNPs that were polymorphic with a $\mathrm{MAF} \geq 0.01$ in Dataset-2 varied from 0.001 to 0.662 , with an overall average of 0.243 (Supplementary Table S6).
Nearly $56 \%$ of the pairs of samples differed by just $<5 \%$ of the scored alleles. All pairs of African rice samples, 50.2\% of the $O$. barthii pairs and 5.2\% of Asian rice pairs differed by $<5 \%$ of the alleles. Approximately $36 \%$ of the pairs of samples differed by more than $50 \%$ of the alleles in Dataset-2 which was due to greater distance among pairs of samples belong to different species. Neighbor-joining tree constructed from the genetic distance matrix of all 3245 samples showed three major groups (Supplementary Fig. S2). The first group consisted of all samples belonging to the African rice and $O$. barthii. The second and third groups consisted of all Asian rice samples adapted to the lowland (which are primarily indica) and the upland (primarily japonica) ecologies in Africa, as summarized in Supplementary Table S1. The first two principal components from PCA performed across all three species accounted for $84.7 \%$ of the molecular variation observed across the 3245 samples. A plot of PC1 (74.3\%) and PC2 (10.4\%) showed clear population structure in the same way as the neighbor-joining analysis (Fig. 1).

We then performed separate analyses on genotypic data of each species to get a better insight into the extent of genetic variation among accessions belonging to each species. Among the 26,073 SNPs in Dataset-1, the number of SNPs that were polymorphic within each species (at a MAF $\geq 0.01$ ) was $10.9 \%$ (2840 SNPs) in African rice, $18.4 \%$ (4807 SNPs) in O. barthii, $54.1 \%$ in lowland Asian rice and $34.0 \%$ in upland Asian rice (Table 1). The proportion of polymorphism in African rice accounted for $59.1 \%, 20.1 \%$ and $32.1 \%$ of the polymorphism observed within $O$. barthii, lowland and upland Asian rice, respectively. Genetic distance estimated from SNPs that were polymorphic within each species/group varied from 0.004 to 0.308 in African rice, from 0.011 to 0.349 in $O$. barthii, from 0.013 to 0.466 in lowland Asian rice and from 0.018 to 0.416 in upland Asian rice (Supplementary Table S7). In African rice, the genetic distance values for about $78 \%$ and $12 \%$ of the pairs of accessions varied from 0.101 to 0.200 and from 0.201 to 0.300 , respectively, with none of the pairs having a genetic distance exceeding 0.308 . On the contrary, approximately $22 \%, 50 \%$ and $24 \%$ of the pairs of $O$. barthii accessions had genetic distance values ranging from 0.101 to 0.200 , from 0.201 to 0.300 and from 0.301 to 0.400 , respectively (Fig. 3, Supplementary Table S7).

The overall average distance observed within African rice $(0.152)$ accounted for $63.1 \%$ and $80.0 \%$ of the average distance observed in $O$. barthii $(0.241)$ and lowland Asian rice (0.190), respectively, but it was $12.6 \%$ greater than that of upland Asian rice (0.135) (Supplementary Table S7). The phylogenetic tree constructed from the genetic distance matrix computed from 3484 SNPs that were polymorphic in both African rice and $O$. barthii showed three major groups, with the African rice samples partitioned into six subgroups (Supplementary Fig. S5). 


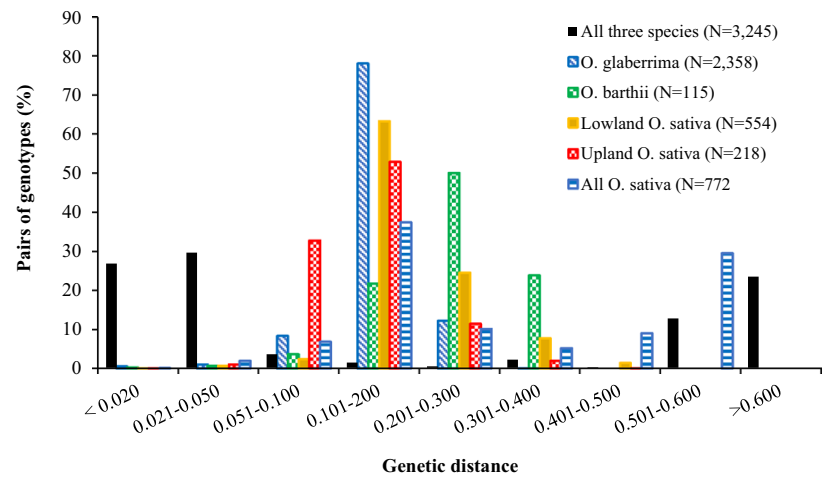

Fig. 3 Genetic distance summaries between pairs of 3245 samples computed from SNPs that were polymorphic across all three species (23,079 SNPs), O. glaberrima (2840 SNPs), O. barthii (4807 SNPs), lowland $O$. sativa (14,106 SNPs) and upland $O$. sativa (8860 SNPs). The number of samples is shown in the legend within brackets

Plots of PC1 and PC2 from PCA (Supplementary Fig. S6) showed similar pattern as the cluster analysis. Detailed results of 2179 of the 2358 (94.4\%) African rice samples have been presented in our previous study (Ndjiondjop et al. 2017). The addition of 179 African rice samples and the inclusion of the $O$. barthii samples changed the overall results only a little. The partitioning of the overall molecular variance (AMOVA) into four hierarchical levels corresponding to African rice, $O$. barthii, lowland Asian rice and upland Asian rice revealed that differences among groups accounted for $92.4 \%$ of the total variation (Table 4). Pairwise $F_{\mathrm{ST}}$ computed for African rice against $O$. barthii, lowland Asian rice and upland Asian rice was $0.276,0.939$ and 0.964 , respectively. $F_{\mathrm{ST}}$ values between $O$. barthii vs. lowland, $O$. barthii vs. upland and lowland vs upland Asian rice were 0.844, 0.919 and 0.0.7389, respectively.

\section{Discussion}

\section{Genetic relatedness, sample sizes and misclassification}

Details comparing genetic distance, relatedness and population structure within African rice and Asian rice have already been presented in our previous studies (Ndjiondjop et al. 2017, 2018a). The primary focus of the present study was to compare marker polymorphism, nucleotide diversity, genetic relatedness and population structure among the three AA-genome rice species using DArTseq-based genomewide SNPs and SNPs that fell within selective sweeps identified in African rice. Polymorphism levels (Table 1), nucleotide diversity (Table 2) and genetic distances (Supplementary Table S7) in African rice were all greatly reduced compared to $O$. barthii, which are consistent with previous studies irrespective of marker types and density (Joshi et al. 2000; Ishii et al. 2001; Park et al. 2003; Semon et al. 2005; Kwon et al. 2006; Li et al. 2011; Orjuela et al. 2014; Wang et al. 2014; Meyer et al. 2016; Ndjiondjop et al. 2017). The lower genetic distance values among most pairs of African rice samples were also evident in both phylogenetic tree (Supplementary Fig. S2) and PCA plot (Fig. 1); these plots of genetic structure and relationships also agree with previous studies (Li et al. 2011; Orjuela et al. 2014; Cubry et al. 2018). Orjuela et al. (2014) found two subgroups for African rice and three subgroups for $O$. barthii primarily based on geography and that African rice and $O$. barthii were closer together than either of them compared to Asian rice. Wang et al. (2014) found five groups and that all $O$. barthii and African rice accessions from Gambia, Guinea, Senegal and Sierra Leone formed two admixture groups (OB-V and OB-IV), whereas the other accessions sampled outside the domestication center formed three additional groups. In the current study, we found six groups of African rice and that the group membership of the $O$. barthii samples generally

Table 4 Analysis of molecular variance (AMOVA) for the extraction of SNP variation among and within groups (populations)

\begin{tabular}{|c|c|c|c|c|c|c|}
\hline Category & $\begin{array}{l}\text { No. of SNPs } \\
\text { used for analysis }\end{array}$ & Source of variation & d.f. & Sum of squares & $\begin{array}{l}\text { Variance } \\
\text { components }\end{array}$ & $\begin{array}{l}\text { Percentage } \\
\text { of variation }\end{array}$ \\
\hline \multirow{3}{*}{$\begin{array}{l}\text { Four groups }(O . \text { glaberrima, } O . \text { barthii, } \\
\text { lowland } O . \text { sativa and upland } O . \text { sativa })\end{array}$} & \multirow[t]{3}{*}{23,079} & Among groups & 3 & $7,600,354.2$ & 5358.1 & 92.8 \\
\hline & & Within groups & 3241 & $1,347,725.3$ & 415.8 & 7.2 \\
\hline & & Total & 3244 & $8,948,079.4$ & 5774.0 & 100.0 \\
\hline \multirow[t]{3}{*}{ Two groups ( $O$. glaberrima and $O$. barthii) } & \multirow[t]{3}{*}{23,079} & Among groups & 1.0 & $18,837.7$ & 84.9 & 27.65 \\
\hline & & Within groups & 2471.0 & $548,912.1$ & 222.1 & 72.35 \\
\hline & & Total & 2472 & $567,749.8$ & 307.0 & 100.0 \\
\hline \multirow[t]{3}{*}{ Two groups ( $O$. glaberrima and $O$. barthii) } & \multirow[t]{3}{*}{3484} & Among groups & 1.0 & $18,466.0$ & 83.2 & 27.67 \\
\hline & & Within groups & 2471.0 & $537,451.2$ & 217.5 & 72.33 \\
\hline & & Total & 2472 & $555,917.2$ & 300.7 & 100.0 \\
\hline
\end{tabular}


agrees with their country of origin but not ecology. However, nearly $40 \%$ of the $O$. barthii accessions were clustered together with African rice accessions, which differed between the current study and other studies (Orjuela et al. 2014; Wang et al. 2014), which may be due to differences in the genetic background of the germplasm and sample size (Iwamoto et al. 1999; Lu et al. 2000; Nagano et al. 2000; Ishii et al. 2001; Park et al. 2003; Ren et al. 2003; Zhu and Ge 2005; Duan et al. 2007; Zhang et al. 2014; Wambugu et al. 2015; Yin et al. 2016).

The close relationship between $O$. barthii and many of the African rice accessions undoubtedly reflects the direct relationship between the two species, but it may also be caused by some misclassification of accessions. In previous studies, we found out that $3.1 \%$ of accessions across four rice species were misclassified/misidentified (Ndjiondjop et al. 2018b), which was smaller than the 4-21\% misclassification reported in other studies (Buso et al. 2001; Girma et al. 2012; Orjuela et al. 2014; Mason et al. 2015). To minimize errors due to misclassification during germplasm collection, acquisitions and routine genebank operations, our group identified 332 species- and subspecies-specific diagnostic SNP markers in $O$. glaberrima/O. barthii, O. sativa spp. indica, $O$. sativa spp. japonica and $O$. longistaminata that can be used for genotyping quality control analysis; however, none of the markers were diagnostic between $O$. glaberrima and $O$. barthii (Ndjiondjop et al. 2018b), which is another indicator of very close genetic relationship between these two species.

We used varying sample sizes, varying marker numbers and three methods to detect genomic regions with an evidence of selective signature during domestication: (1) the identification of selective sweeps, (2) the genetic diversity ratio of the wild and cultivated species and (3) the identification of loci that had undergone extreme genetic differentiation (Cubry et al. 2018), which all revealed consistently much smaller genetic variation in African rice than its wild progenitor $O$. barthii and Asian rice, irrespective of sample size and marker density. The reduction in genetic diversity in African rice as compared to its wild progenitor was consistent with previous studies conducted on smaller number of samples ranging from 19 to 163 accessions, and with molecular markers or whole genome sequencing ( $\mathrm{Li}$ et al. 2011; Wang et al. 2014; Meyer et al. 2016; Win et al. 2017; Cubry et al. 2018).

\section{Selective sweeps in African rice}

A severe reduction in nucleotide diversity was observed across diverse sample size of African rice when 780 SNPs in Dataset-3 that mapped within the 37 candidate selective sweep regions detected in this species were used for analysis compared to those detected in $O$. barthii and Asian rice (Table 2). Such sharp reduction in nucleotide diversity in
African rice in the selective sweeps regions is likely due to positive selection during and/or after domestication as compared with the other two species, as has been reported around known rice domestication genes (Li et al. 2006, 2011; Olsen et al. 2006; Jin et al. 2008; Zhang et al. 2009; He et al. 2011; Zhu et al. 2011; Huang et al. 2012; Hua et al. 2015; Civáň and Brown 2017; Win et al. 2017; Lv et al. 2018). Some of the most widely cited domestication genes reported in African and Asian rice include the semidwarf gene (SD1) (Cho et al. 1994) and a major effect QTL for grain shattering (qSH1) that accounted for 36\% of yield difference between indica and japonica cultivars (Konishi et al. 2006; Onishi et al. 2007) on chromosome 1; long kernel 3 (LK3) or grain size 3 (GS3) (Fan et al. 2006; Takano-Kai et al. 2009) on chromosome 3; SHAT1, which encodes APETALA2 (AP2) transcription factor (Zhou et al. 2012), shattering 3 (Sh3), shattering 4 (Sh4) (Inoue et al. 2015; Wu et al. 2017), hull color (Bh4) (Zhu et al. 2011; Vigueira et al. 2013) and LONG AND BARBED AWN1 (LABA1) (Hua et al. 2015) genes on chromosome 4; SHATTERING 5 (SH5) and a major effect grain width QTL (qSW5/GW5) on chromosome 5; SHATTERING-H, Red grain color (Rc) and Prostrate growth 1 (PROG1), which controls the transition from prostate to erect growth habit (Tan et al. 2008) on chromosome 7.

Although we were not confidently able to locate the same domestication genes in our study, we found various candidate genes with known functions that mapped within the 37 selective sweep regions detected in African rice (Supplementary Tables S3, S4). Some of the candidate genes that fell within the selective sweep region on chromosome 9 with the highest Omega scores were a Jasmonate ZIMdomain protein that induces resistance to bacterial blight (Yamada et al. 2012); DEEPER ROOTING 1 (DRO1) that controls root system architecture and drought avoidance and increases grain yield under drought conditions (Uga et al. 2013); ALDEHYDE DEHYDROGENASE 7, which is required for seed maturation and maintenance of viability during storage (Shin et al. 2009); DELAYED SEED GERMINATION 1 (OsDSG1) that controls seed germination in storage and stress responses in rice (Park et al. 2010); ETHYLENE RESPONSE FACTOR 72, which regulates expression of a wide variety of downstream target genes related to stress response and development (Phukan et al. 2017) and CELLULOSE SYNTHASE-LIKE C2 protein, which is required for cellulose synthesis and larger growth $(\mathrm{Gu}$ and Somerville 2010). Other genes of known function that fell within the second selective sweep on chromosome 9 (9: 6,267,139-6,508,307 bp) are SUBMERGENCE 1B (Sub1B) and Sub1C, which are involved in rice tolerance to submergence (Fukao et al. 2006), and NA+/H+ANTIPORTER 5, which is a sodium/hydrogen exchanger subfamily protein that enhances salinity tolerance in rice (Verma et al. 2007; 
Khan 2011), both of which are needed when rice is grown under flood irrigation.

The second highest Omega scores were observed for a selective sweep region identified on chromosome 11, which spans $153 \mathrm{~kb}(11: 16,030,588-16,183,929 \mathrm{bp})$ and harbors 5 candidate genes (Supplementary Tables S3, S4). The latter includes an aminotransferase-like enzyme, which is involved in a number of metabolic activities, including abiotic stress in rice (Kothari et al. 2016); a Bx2-like protein that is associated with iron toxicity in rice (Finatto et al. 2015) and protein kinase domain containing protein that catalyze the transfer of the phosphate from nucleotide triphosphates to one or more amino acid residues in a protein substrate side chain. Of the candidate genes that mapped within the $695 \mathrm{~kb}$ interval on chromosome 2 (2: 5,973,055-6,668,406), PLASTIDIC NUCLEOTIDE TRANSPORT PROTEIN is involved in the carbon flow related to starch metabolism and thus larger, more nutritious grain (Toyota et al. 2006); RECEPTOR-LIKE CYTOPLASMIC KINASE plays a role in plant signaling (Liang and Zhou 2018); PLANT DEFENSIN 1.2 is involved in defense against fungi (Silverstein et al. 2005); CLASS-1-TYPE HISTONE DEACETYLASE is involved in reproductive development, seed morphology and plant architecture (Jang et al. 2003); gamma ray-induced Leucinerich repeat receptor-like kinase (LRR-RLK) plays key roles in abiotic stresses tolerance (Park et al. 2014); ALLENE OXIDE SYNTHASE 3 plays a role in the biosynthesis of jasmonic acid, while $\beta$-galactosidase is involved in plant defense and the metabolism of galactose-rich polymers (Esteban et al. 2003).

\section{Conclusion}

The present study was conducted on a large collection of genotypes representing the three AA-genome Oryza species genotyped using DArTseq-based genotyping by sequencing. Our study clearly demonstrated a narrowing of genetic diversity within the cultivated African rice as compared with its wild progenitor. The reduction in the overall nucleotide diversity in African rice was 14-20-fold (depending on sample size) when the analyses were conducted on the genotype data of 780 SNPs that fell within 37 selective sweep regions identified in this species. These regions contained various annotated genes other than the well-known domestication genes whose functions provide additional clues as to how domestication proceeded from $O$. barthii to cultivated African rice.

Author contribution statement MNN conceived, designed and supervised the experiments, secured funding and partly drafted the paper; AG and SBK were responsible for sample preparation, DNA extraction and/or compilation of passport information; NA conducted the selective sweep analyses; KS conducted most analyses and wrote most part of the paper; DZ and PP contributed to and edited the paper. All authors read and approved the paper.

Acknowledgements The authors would like to thank Elisée Dannon, Marie Gouette and Blandine Fatondji for assisting on samples preparation and DNA extraction and Dr Marilyn L. Warburton (Corn Host Plant Resistance Research Unit, USDA-Agricultural Research Service, Mississippi State, MS, USA) for editing the paper. The present study was supported by a grant given to $\mathrm{M}$. Ndjiondjop (AfricaRice) from the Global Diversity Crop Trust (GDCT) through CGIAR Systems Organization and by the Federal Ministry for Economic Cooperation and Development, Germany.

\section{Compliance with ethical standards}

Conflict of interest On behalf of all authors, the corresponding author states that there is no conflict of interest.

Ethical approval Seed samples were obtained from the AfricaRice genebank, Cote d'Ivoire. All accessions/genotypes used in this study are public goods and freely available for non-commercial purpose.

Availability of data and materials All relevant files are included within this article and its supplementary files. The raw genotype data of all accessions will be deposited in public database upon acceptance of the manuscript.

Open Access This article is distributed under the terms of the Creative Commons Attribution 4.0 International License (http://creativeco mmons.org/licenses/by/4.0/), which permits unrestricted use, distribution, and reproduction in any medium, provided you give appropriate credit to the original author(s) and the source, provide a link to the Creative Commons license, and indicate if changes were made.

\section{References}

Alachiotis N, Pavlidis P (2016) Scalable linkage-disequilibrium-based selective sweep detection: a performance guide. GigaScience 5:7

Alachiotis N, Pavlidis P (2018) RAiSD detects positive selection based on multiple signatures of a selective sweep and SNP vectors. Commun Biol 1:79

Alachiotis N, Stamatakis A, Pavlidis P (2012) OmegaPlus: a scalable tool for rapid detection of selective sweeps in whole-genome datasets. Bioinformatics 28:2274-2275

Buso GSC, Rangel PHN, Ferreira ME (2001) Analysis of random and specific sequences of nuclear and cytoplasmic DNA in diploid and tetraploid American wild rice species (Oryza spp.). Genome 44:476-494

Chen M, Pan D, Ren H, Fu J, Li J, Su G, Wang A, Jiang L, Zhang Q, Liu J-F (2016) Identification of selective sweeps reveals divergent selection between Chinese Holstein and Simmental cattle populations. Genet Sel Evol 48:76

Cho Y, Eun M, Kim Y, Chung T, Chae Y (1994) The semidwarf gene, sd-1, of rice (Oryza sativa L.). I. Linkage with the esterase locus, Estl-2. Theor Appl Genet 89:49-53

Civáň P, Brown TA (2017) Origin of rice (Oryza sativa L.) domestication genes. Genet Resour Crop Evol 64:1125-1132 
Crisci JL, Poh Y-P, Mahajan S, Jensen JD (2013) The impact of equilibrium assumptions on tests of selection. Front Genet 4:235

Cubry P, Tranchant-Dubreuil C, Thuillet A-C, Monat C, Ndjiondjop M-N, Labadie K, Cruaud C, Engelen S, Scarcelli N, Rhoné B, Burgarella C, Dupuy C, Larmande P, Wincker P, François O, Sabot F, Vigouroux Y (2018) The rise and fall of African rice cultivation revealed by analysis of 246 new genomes. Curr Biol 28:2274-2282

Ding Z, Wang C, Chen S, Yu S (2011) Diversity and selective sweep in the OsAMT1; 1 genomic region of rice. BMC Evol Biol 11:61

Duan S, Lu B, Li Z, Tong J, Kong J, Yao W, Li S, Zhu Y (2007) Phylogenetic analysis of AA-genome Oryza species (poaceae) based on chloroplast, mitochondrial, and nuclear DNA sequences. Biochem Genet 45:113-129

Esteban R, Dopico B, Muñoz FJ, Romo S, Martín I, Labrador E (2003) Cloning of a Cicer arietinum $\beta$-Galactosidase with pectin-degrading function. Plant Cell Physiol 44:718-725

Excoffier L, Lischer HEL (2010) Arlequin suite ver 3.5: a new series of programs to perform population genetics analyses under Linux and Windows [electronic resource]. Mol Ecol Resour 10:564-567

Excoffier L, Smouse PE, Quattro JM (1992) Analysis of molecular variance inferred from metric distances among DNA haplotypes: application to human mitochondrial DNA restriction data. Genetics 131:479-491

Fan C, Xing Y, Mao H, Lu T, Han B, Xu C, Li X, Zhang Q (2006) GS3, a major QTL for grain length and weight and minor QTL for grain width and thickness in rice, encodes a putative transmembrane protein. Theor Appl Genet 112:1164-1171

Finatto T, de Oliveira AC, Chaparro C, da Maia LC, Farias DR, Woyann LG, Mistura CC, Soares-Bresolin AP, Llauro C, Panaud O, Picault N (2015) Abiotic stress and genome dynamics: specific genes and transposable elements response to iron excess in rice. Rice 8:13

Fukao T, Xu K, Ronald PC, Bailey-Serres J (2006) A variable cluster of ethylene response factor-like genes regulates metabolic and developmental acclimation responses to submergence in rice. Plant Cell 18:2021

Girma G, Korie S, Dumet D, Franco J (2012) Improvement of accession distinctiveness as an added value to the global worth of the yam (Dioscorea spp.) genebank. Int J Conserv Sci 3:199-206

Gore MA, Chia JM, Elshire RJ, Sun Q, Ersoz ES, Hurwitz BL, Peiffer JA, McMullen MD, Grills GS, Ross-Ibarra J, Ware DH, Buckler ES (2009) A first-generation haplotype map of maize. Science 326:1115-1117

Gu Y, Somerville C (2010) Cellulose synthase interacting protein: a new factor in cellulose synthesis. Plant Signal Behav 5:1571-1574

He Z, Zhai W, Wen H, Tang T, Wang Y, Lu X, Greenberg AJ, Hudson RR, Wu CI, Shi S (2011) Two evolutionary histories in the genome of rice: the roles of domestication genes. PLoS Genet 7:e1002100

Holsinger KE, Weir BS (2009) Genetics in geographically structured populations: defining, estimating and interpreting FST. Nature Rev Genet 10:639-650

Hua L, Wang DR, Tan L, Fu Y, Liu F, Xiao L, Zhu Z, Fu Q, Sun X, Gu P, Cai H, McCouch SR, Sun C (2015) LABA1, a domestication gene associated with long, barbed awns in wild rice. Plant Cell $27: 1875-1888$

Huang X, Kurata N, Wei X, Wang Z-X, Wang A, Zhao Q, Zhao Y, Liu K, Lu H, Li W, Guo Y, Lu Y, Zhou C, Fan D, Weng Q, Zhu C, Huang T, Zhang L, Wang Y, Feng L (2012) A map of rice genome variation reveals the origin of cultivated rice. Nature 490:497-501

Inoue C, Htun TM, Inoue K, Ikeda KI, Ishii T, Ishikawa R (2015) Inhibition of abscission layer formation by an interactionof two seedshattering loci, sh4 and qSH3, in rice. Genes Genet Syst 90:1-9

Ishii T, Xu Y, McCouch SR (2001) Nuclear- and chloroplast-microsatellite variation in A-genome species of rice. Genome 44:658-666
Iwamoto M, Nagashima H, Nagamine T, Higo H, Higo K (1999) p-SINE1-like intron of the CatA catalase homologs and phylogenetic relationships among AA-genome Oryza and related species. Theor Appl Genet 98:853-861

Jang I-C, Pahk Y-M, Song SI, Kwon HJ, Nahm BH, Kim J-K (2003) Structure and expression of the rice class-I type histone deacetylase genes OsHDAC1-3: OsHDAC1 overexpression in transgenic plants leads to increased growth rate and altered architecture. Plant J 33:531-541

Jin J, Huang W, Gao JP, Yang J, Shi M, Zhu MZ, Luo D, Lin HX (2008) Genetic control of rice plant architecture under domestication. Nat Genet 40:1365-1369

Joshi SP, Gupta VS, Aggarwal RK, Ranjekar PK, Brar DS (2000) Genetic diversity and phylogenetic relationship as revealed by inter simple sequence repeat (ISSR) polymorphism in the genus Oryza. Theor Appl Genet 100:1311-1320

Khan MS (2011) Role of sodium and hydrogen $(\mathrm{Na}+\mathrm{H}+)$ antiporters in salt tolerance of plants: present and future challenges. Afr J Biotechnol 10:13693-13704

Konishi S, Izawa T, Lin SY, Ebana K, Fukuta Y, Sasaki T, Yano M (2006) An SNP caused loss of seed shattering during rice domestication. Science 312:1392-1396

Kothari KS, Dansana PK, Giri J, Tyagi AK (2016) Rice stress associated protein 1 (OsSAP1) interacts with aminotransferase (OsAMTR1) and pathogenesis-related 1a protein (OsSCP) and regulates abiotic stress responses. Front Plant Sci 7:1057

Kumar S, Stecher G, Tamura K (2016) MEGA7: molecular evolutionary genetics analysis version 7.0 for bigger datasets. Mol Biol Evol 33:1870-1874

Kwon SJ, Ju KL, Hong SW, Park YJ, McNally KL, Kim NS (2006) Genetic diversity and phylogenetic relationship in AA Oryza species as revealed by Rim2/Hipa CACTA transposon display. Genes Genet Syst 81:93-101

Lam H-M, Xu X, Liu X, Chen W, Yang G, Wong F-L, Li M-W, He W, Qin N, Wang B, Li J, Jian M, Wang J, Shao G, Wang J, Sun SS-M, Zhang G (2010) Resequencing of 31 wild and cultivated soybean genomes identifies patterns of genetic diversity and selection. Nat Genet 42:1053-1059

Li C, Zhou A, Sang T (2006) Rice domestication by reducing shattering. Science 311:1936

Li ZM, Zheng XM, Ge S (2011) Genetic diversity and domestication history of African rice (Oryza glaberrima) as inferred from multiple gene sequences. Theor Appl Genet 123:21-31

Liang X, Zhou J-M (2018) Receptor-like cytoplasmic kinases: central players in plant receptor kinase-mediated signaling. Annu Rev Plant Biol 69:267-299

Lischer HEL, Excoffier L (2012) PGDSpider: an automated data conversion tool for connecting population genetics and genomics programs. Bioinformatics 28:298-299

Lu BR, Naredo MEB, Juliano AB, Jackson MT (2000) Preliminary studies on taxonomy and biosystematics of the AA genome Oryza species (Poaceae). In: Jacobs SWL, Everett J (eds) Grasses, systematics and evolution. CSIRO, Melbourne

Lv S, Wu W, Wang M, Meyer RS, Ndjiondjop M-N, Tan L, Zhou H, Zhang J, Fu Y, Cai H, Sun C, Wing RA, Zhu Z (2018) Genetic control of seed shattering during African rice domestication. Nat Plants 4:331-337

Mason AS, Zhang J, Tollenaere R, Vasquez Teuber P, Dalton-Morgan J, Hu L, Yan G, Edwards D, Redden R, Batley J (2015) High-throughput genotyping for species identification and diversity assessment in germplasm collections. Mol Ecol Resour 15:1091-1101

Meyer RS, Choi JY, Sanches M, Plessis A, Flowers JM, Amas J, Dorph K, Barretto A, Gross B, Fuller DQ, Bimpong IK, Ndjiondjop M-N, Hazzouri KM, Gregorio GB, Purugganan MD (2016) Domestication history and geographical 
adaptation inferred from a SNP map of African rice. Nat Genet 48:1083-1088

Nabholz B, Sarah G, Sabot F, Ruiz M, Adam H, Nidelet S, Ghesquière A, Santoni S, David J, Glémin S (2014) Transcriptome population genomics reveals severe bottleneck and domestication cost in the African rice (Oryza glaberrima). Mol Ecol 23:2210-2227

Nagano H, Kawasaki S, Kishima Y, Sano Y (2000) Structural differences in the vicinity of the waxy locus among the Oryza species with the AA-genome: identification of variable regions. Theor Appl Genet 100:376-383

Ndjiondjop M-N, Semagn K, Gouda AC, Kpeki SB, Dro Tia D, Sow M, Goungoulou A, Sie M, Perrier X, Ghesquiere A, Warburton ML (2017) Genetic variation and population structure of Oryza glaberrima and development of a mini-core collection using DArTseq. Front Plant Sci 8:1748

Ndjiondjop MN, Semagn K, Sow M, Manneh B, Gouda AC, Kpeki SB, Pegalepo E, Wambugu P, Sie M, Warburton ML (2018a) Assessment of genetic variation and population structure of diverse rice genotypes adapted to lowland and upland ecologies in Africa using SNPs. Front Plant Sci 9:446

Ndjiondjop MN, Semagn K, Zhang J, Gouda AC, Kpeki SB, Goungoulou A, Wambugu P, Dramé KN, Bimpong IK, Zhao D (2018b) Development of species diagnostic SNP markers for quality control genotyping in four rice (Oryza L.) species. Mol Breed 38:131

Nielsen R, Williamson S, Kim Y, Hubisz MJ, Clark AG, Bustamante C (2005) Genomic scans for selective sweeps using SNP data. Genome Res 15:1566-1575

Olsen KM, Caicedo AL, Polato N, McClung A, McCouch S, Purugganan MD (2006) Selection under domestication: evidence for a sweep in the rice waxy genomic region. Genetics 173:975-983

Onishi K, Takagi K, Kontani M, Tanaka T, Sano Y (2007) Different patterns of genealogical relationships found in the two major QTLs causing reduction of seed shattering during rice domestication. Genome 50:757-766

Orjuela J, Sabot F, Chéron S, Vigouroux Y, Adam H, Chrestin H, Sanni K, Lorieux M, Ghesquière A (2014) An extensive analysis of the African rice genetic diversity through a global genotyping. Theor Appl Genet 127:2211-2223

Park KC, Kim NH, Cho YS, Kang KH, Lee JK, Kim NS (2003) Genetic variations of AA genome Oryza species measured by MITEAFLP. Theor Appl Genet 107:203-209

Park G-G, Park J-J, Yoon J, Yu S-N, An G (2010) A RING finger E3 ligase gene, Oryza sativa delayed seed germination 1 (OsDSG1), controls seed germination and stress responses in rice. Plant Mol Biol 74:467-478

Park S, Moon J-C, Park YC, Kim J-H, Kim DS, Jang CS (2014) Molecular dissection of the response of a rice leucine-rich repeat receptor-like kinase (LRR-RLK) gene to abiotic stresses. J Plant Physiol 171:1645-1653

Pavlidis P, Alachiotis N (2017) A survey of methods and tools to detect recent and strong positive selection. J Biol Res Thessaloniki 24:7

Pavlidis P, Živković D, Stamatakis A, Alachiotis N (2013) SweeD: likelihood-based detection of selective sweeps in thousands of genomes. Mol Biol Evol 30:2224-2234

Peter BM, Huerta-Sanchez E, Nielsen R (2012) Distinguishing between selective sweeps from standing variation and from a de novo mutation. PLoS Genet 8:e1003011

Phukan UJ, Jeena GS, Tripathi V, Shukla RK (2017) Regulation of Apetala2/Ethylene response factors in plants. Front Plant Sci $8: 150$

Reif JC, Zhang P, Dreisigacker S, Warburton ML, van Ginkel M, Hoisington D, Bohn M, Melchinger AE (2005) Wheat genetic diversity trends during domestication and breeding. Theor Appl Genet 110:859-864
Ren F, Lu BR, Li S, Huang J, Zhu Y (2003) A comparative study of genetic relationships among the AA-genome Oryza species using RAPD and SSR markers. Theor Appl Genet 108:113-120

Semon M, Nielsen R, Jones MP, McCouch SR (2005) The population structure of African cultivated rice Oryza glaberrima (Steud.): evidence for elevated levels of linkage disequilibrium caused by admixture with $O$. sativa and ecological adaptation. Genetics 169:1639-1647

Shin J-H, Kim S-R, An G (2009) Rice aldehyde dehydrogenase7 is needed for seed maturation and viability. Plant Physiol 149:905

Silverstein KAT, Graham MA, Paape TD, VandenBosch KA (2005) Genome organization of more than 300 defensin-like genes in Arabidopsis. Plant Physiol 138:600-610

Stein JC, Yu Y, Copetti D, Zwickl DJ, Zhang L, Zhang C, Chougule K, Gao D, Iwata A, Goicoechea JL, Wei S, Wang J, Liao Y, Wang M, Jacquemin J, Becker C, Kudrna D, Zhang J, Londono CEM, Song X, Lee S, Sanchez P, Zuccolo A, Ammiraju JSS, Talag J, Danowitz A, Rivera LF, Gschwend AR, Noutsos C, Wu CC, Kao SM, Zeng JW, Wei FJ, Zhao Q, Feng Q, El Baidouri M, Carpentier MC, Lasserre E, Cooke R, Rosa Farias DD, Da Maia LC, Dos Santos RS, Nyberg KG, McNally KL, Mauleon R, Alexandrov N, Schmutz J, Flowers D, Fan C, Weigel D, Jena KK, Wicker T, Chen M, Han B, Henry R, Hsing YIC, Kurata N, De Oliveira AC, Panaud O, Jackson SA, Machado CA, Sanderson MJ, Long M, Ware D, Wing RA (2018) Genomes of 13 domesticated and wild rice relatives highlight genetic conservation, turnover and innovation across the genus Oryza. Nat Genet 50:285-296

Tajima F (1989) Statistical method for testing the neutral mutation hypothesis by DNA polymorphism. Genetics 123:585

Takano-Kai N, Jiang H, Kubo T, Sweeney M, Matsumoto T, Kanamori H, Padhukasahasram B, Bustamante C, Yoshimura A, Doi K, McCouch S (2009) Evolutionary history of GS3, a gene conferring grain length in rice. Genetics 182:1323-1334

Tan L, Li X, Liu F, Sun X, Li C, Zhu Z, Fu Y, Cai H, Wang X, Xie D, Sun C (2008) Control of a key transition from prostrate to erect growth in rice domestication. Nat Genet 40:1360

Toyota K, Tamura M, Ohdan T, Nakamura Y (2006) Expression profiling of starch metabolism-related plastidic translocator genes in rice. Planta 223:248-257

Uga Y, Sugimoto K, Ogawa S, Rane J, Ishitani M, Hara N, Kitomi Y, Inukai Y, Ono K, Kanno N, Inoue H, Takehisa H, Motoyama R, Nagamura Y, Wu J, Matsumoto T, Takai T, Okuno K, Yano M (2013) Control of root system architecture by DEEPER ROOTING 1 increases rice yield under drought conditions. Nat Genet 45:1097

Vaughan DA, Morishima H, Kadowaki K (2003) Diversity in the Oryza genus. Curr Opin Plant Biol 6:139-146

Vaughan DA, Lu BR, Tomooka N (2008) The evolving story of rice evolution. Plant Sci 174:394-408

Verma D, Singla-Pareek SL, Rajagopal D, Reddy MK, Sopory SK (2007) Functional validation of a novel isoform of $\mathrm{Na}+\mathrm{H}+$ antiporter from Pennisetum glaucum for enhancing salinity tolerance in rice. J Biosci (Bangalore) 32:621-628

Vigueira CC, Li W, Olsen KM (2013) The role of Bh4 in parallel evolution of hull colour in domesticated and weedy rice. J Evol Biol 26:1738-1749

Vitti JJ, Grossman SR, Sabeti PC (2013) Detecting natural selection in genomic data. Annu Rev Genet 47:97-120

Voight BF, Kudaravalli S, Wen X, Pritchard JK (2006) A map of recent positive selection in the human genome. PLoS Biol 4:e72

Wambugu PW, Brozynska M, Furtado A, Waters DL, Henry RJ (2015) Relationships of wild and domesticated rices (Oryza AA genome species) based upon whole chloroplast genome sequences. Sci Rep 5:13957 
Wang MH, Yu YS, Haberer G, Marri PR, Fan CZ, Goicoechea JL, Zuccolo A, Song X, Kudrna D, Ammiraju JSS, Cossu RM, Maldonado C, Chen J, Lee SH, Sisneros N, de Baynast K, Golser W, Wissotski M, Kim WJ, Sanchez P, Ndjiondjop MN, Sanni K, Long MY, Carney J, Panaud O, Wicker T (2014) The genome sequence of African rice (Oryza glaberrima) and evidence for independent domestication. Nat Genet 46:982-988

Win KT, Yamagata Y, Doi K, Uyama K, Nagai Y, Toda Y, Kani T, Ashikari M, Yasui H, Yoshimura A (2017) A single base change explains the independent origin of and selection for the nonshattering gene in African rice domestication. New Phytol 213:1925-1935

Wright SI, Bi IV, Schroeder SG, Yamasaki M, Doebley JF, McMullen MD, Gaut BS (2005) The effects of artificial selection on the maize genome. Science 308:1310-1314

Wu W, Liu X, Wang M, Meyer RS, Luo X, Ndjiondjop M-N, Tan L, Zhang J, Wu J, Cai H, Sun C, Wang X, Wing RA, Zhu Z (2017) A single-nucleotide polymorphism causes smaller grain size and loss of seed shattering during African rice domestication. Nat Plants 3:17064

Yamada S, Kano A, Tamaoki D, Miyamoto A, Shishido H, Miyoshi S, Taniguchi S, Akimitsu K, Gomi K (2012) Involvement of OsJAZ8 in jasmonate-induced resistance to bacterial blight in rice. Plant Cell Physiol 53:2060-2072

Yin H, Akimoto M, Kaewcheenchai R, Sotowa M, Ishii T, Ishikawa $\mathrm{R}$ (2016) Inconsistent diversities between nuclear and plastid genomes of AA genome species in the genus oryza. Genes Genet Syst 90:269-281

Yuan Y, Zhang Q, Zeng S, Gu L, Si W, Zhang X, Tian D, Yang S, Wang L (2017) Selective sweep with significant positive selection serves as the driving force for the differentiation of japonica and indica rice cultivars. BMC Genom 18:307

Zhang LB, Zhu Q, Wu ZQ, Ross-Ibarra J, Gaut BS, Ge S, Sang T (2009) Selection on grain shattering genes and rates of rice domestication. New Phytol 184:708-720

Zhang Q-J, Zhu T, Xia E-H, Shi C, Liu Y-L, Zhang Y, Liu Y, Jiang W-K, Zhao Y-J, Mao S-Y, Zhang L-P, Huang H, Jiao J-Y, Xu P-Z, Yao Q-Y, Zeng F-C, Yang L-L, Gao J, Tao D-Y, Wang Y-J, Bennetzen JL, Gao L-Z (2014) Rapid diversification of five Oryza AA genomes associated with rice adaptation. Proc Natl Acad Sci 111:E4954

Zhou Y, Lu D, Li C, Luo J, Zhu B-F, Zhu J, Shangguan Y, Wang Z, Sang T, Zhou B, Han B (2012) Genetic control of seed shattering in rice by the APETALA2 transcription factor SHATTERING ABORTION1. Plant Cell 24:1034-1048

Zhu Q, Ge S (2005) Phylogenetic relationships among A-genome species of the genus Oryza revealed by intron sequences of four nuclear genes. New Phytol 167:249-265

Zhu BF, Si L, Wang Z, Zhou Y, Zhu J, Shangguan Y, Lu D, Fan D, Li C, Lin H, Qian Q, Sang T, Zhou B, Minobe Y, Han B (2011) Genetic control of a transition from black to straw-white seed hull in rice domestication. Plant Physiol 155:1301-1311

Publisher's Note Springer Nature remains neutral with regard to jurisdictional claims in published maps and institutional affiliations. 